\title{
Fasciola hepatica: Sonographische und computertomographische Veränderungen bei Befall mit einem Leberegel
}

Fasciola hepatica (großer Leberegel) ist ein ubiquitärer Parasit von Rindern und Schafen, der nur gelegentlich den Menschen befällt. Seit 1980 ist die Anzahl der menschlichen Infektionen signifikant gestiegen und einige Regionen in der Welt sind bereits endemisch für die humane Infektion dieses primär zoonotischen Parasiten (Mas-Coma et al., Bull World Health Organ 1999; 77: 340).

Die klinischen Symptome ähneln anderen Leber- und Gallenwegserkrankungen. In den bildgebenden Verfahren zeigen sich typische, wenig bekannte Veränderungen, die leicht fehlinterpretiert werden können.

Wir berichten über den seltenen Fall einer Fasciolose beim Menschen mit mechanischer Obstruktion durch den Parasiten, konsekutiver Stauung der intrahepatischen Gallenwege sowie sonographischer und computertomographischer Darstellung des Wurmes in den Gallenwegen.

\section{Fallbeschreibung}

Eine 44-jährige Patientin wurde aufgrund kolikartiger Schmerzen im rechten Oberbauch mit Ausstrahlung in den Rücken, verbunden mit Übelkeit und Erbrechen, zur weiteren Abklärung stationär aufgenommen. Die körperliche Untersuchung war bis auf einen mäßigen Druckschmerz im Epigastrium unauffällig, die Körpertemperatur auf $38^{\circ} \mathrm{C}$ erhöht. Die Labordiagnostik zeigte erhöhte Entzündungsparameter (BSG $16 \mathrm{~mm} / \mathrm{h}$ [Norm < $10 \mathrm{~mm} / \mathrm{h}$ ], CRP 4,4 mg/dl [Norm $<0,5 \mathrm{mg} / \mathrm{dl}]$, Leukozyten $10800 / \mu \mathrm{l}$ [Norm 4000-10000/ $\mu 1]$ ), Cholestaseparameter und erhöhte Serumtransaminasen (Gesamt-Bilirubin 2,0 mg/dl [Norm <1,1 mg/dl], AP $215 \mathrm{U} / \mathrm{l}$ [Norm 60 - $190 \mathrm{U} / 1]$, GOT $93 \mathrm{U} / 1$ [Norm < $19 \mathrm{U} / \mathrm{l}]$, GPT 55 U/1 [Norm < 24 U/l], Gamma-GT $178 \mathrm{U} / 1$ [Norm $28 \mathrm{U} / \mathrm{l}]$ ) sowie eine Eosinophilie von $8 \%$ [Norm < 4\%]. Anamnestisch bestand bei der Patientin keine Vorerkrankung, sie berichtete jedoch von mehrmaligen Urlaubsaufenthalten in der Türkei im letzten Jahr.
Sonographisch zeigten sich eine mäßiggradige Stauung der intrahepatischen Gallenwege und ein auf 1,5 cm Durchmesser erweiterter proximaler Ductus hepatocholedochus mit einem Kalibersprung auf $0,8 \mathrm{~cm}$ im distalen Drittel. In Höhe der Kaliberschwankung konnte intraduktal eine unscharf abgrenzbare, echoreiche tubuläre Struktur von ca. 0,5 cm Durchmesser abgegrenzt werden.

Zur weiteren Abklärung erfolgte eine Computertomographie: Eine ca. $2 \mathrm{~cm}$ lange, intraluminale hyperdense Struktur (ca. $70 \mathrm{HU}$ ) im distalen Ductus choledochus (Abb.1a) mit konsekutiver Erweiterung der proximalen Gallenwege konnte dargestellt werden (Abb.1b). Zusätzlich zeigten sich im Lebersegment 6 mehrere kleine gruppierte hypodense

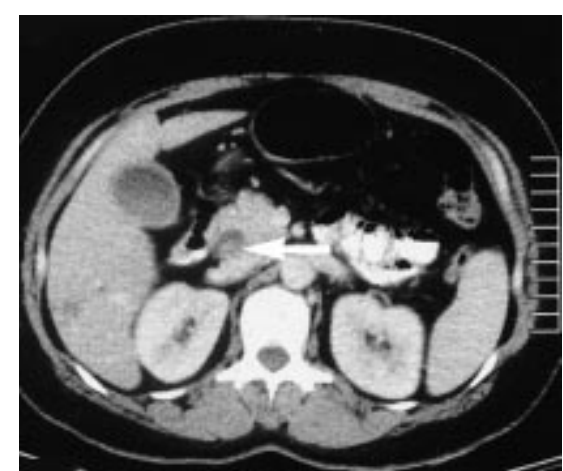

Abb. 1 a Kontrastverstärkte Computertomographie: im distalen Ductus choledochus nachweisbarer Parasit F. hepatica (Pfeil). Beachte die typischen hypodensen Leberläsionen im Segment 6.

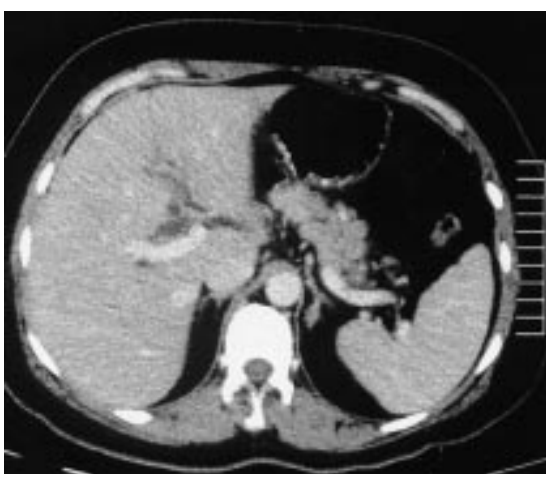

Abb.1 b Erweiterte proximale intrahepatische Gallenwege. 
Läsionen, die straßenförmig angeordnet waren (Abb.1 a).

Die differentialdiagnostische Einordnung des Befundes fiel schwer, primär gingen wir von einer Choledocholithiasis oder einem cholangiozellulären Karzinom aus. In der anschließend durchgeführten ERCP misslang die Sondierung des Ductus choledochus aufgrund einer distalen Stenosierung.

Aufgrund der cholangitischen Zeichen (Fieber, Leukozytose und Erhöhung der Cholestaseparameter) entschloss man sich zu einer PTCD, die ebenfalls die konzentrisch spitz zulaufende Stenose des Ductus choledochus zeigte. Eine Drainage wurde eingelegt.

In der Kontrollsonographie konnte die vorbeschriebene längliche echoreiche Struktur im Ductus choledochus nicht mehr nachgewiesen werden. Stattdessen fand sich eine neu aufgetretene bandartige Struktur ähnlicher Größe und Morphologie am Boden der Gallenblase, als sei die initial beobachtete Struktur nach den Manipulationen gewandert (Abb. 2).

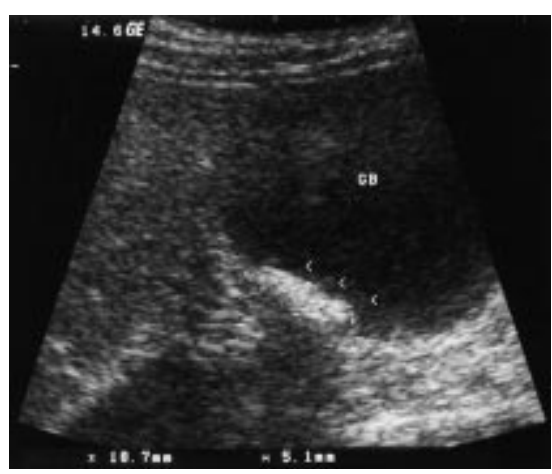

Abb. 2 Sonographie der Gallenblase: der Parasit kommt als echoreiche Struktur am Boden der Gallenblase zur Darstellung.

Aufgrund der bestehenden Eosinophilie erfolgte die Analyse der Gallenflüssigkeit aus der liegenden Drainage im Hinblick auf Parasiten, hier konnten F.-hepaticaEier nachgewiesen werden: Unsere Patientin war somit an einer Fasciolose erkrankt.

Nach einmaliger oraler Therapie mit $750 \mathrm{mg}$ Triclabendazol (Fasinex) konnte die Patientin nach 5 Tagen bei Wohlbefinden entlassen werden.
Die Kontrolluntersuchungen zeigten eine Normalisierung der Laborparameter, einen Rückgang der Eosinophilie und der initial deutlich erhöhten Antikörper gegen F. hepatica sowie eine komplette Rückbildung der sonographischen Befunde. Nach 10 Monaten war die Kontroll-CT bis auf eine angedeutete Hypodensität im Lebersegment 6 unauffällig.

\section{Diskussion}

Infektionen des Menschen mit F. hepatica sind selten. Meist handelt es sich um Einzelfälle, seltener um Epidemien oder ein endemisches Vorkommen.

F. hepatica ist ein $2-4 \mathrm{~cm}$ langer weltweit verbreiteter Saugwurm (Trematodenart), der hauptsächlich Rinder und Schafe befällt. Die Anzahl der menschlichen Infektionen ist in den letzten Jahrzehnten stetig angestiegen und in einigen Regionen der Welt, wie z.B. Bolivien, sind humane Infektionen bereits endemisch. Die Hauptinfektionsquelle für den Menschen stellt der Verzehr von Salaten wild wachsender Pflanzen, vor allem Wasserkresse und Löwenzahn dar.

In der akuten Phase der parenchymalen Leberinvasion durch den Wurm können computertomographisch subkapsulär hypodense Leberareale gezeigt werden. Kernspintomographisch kann die hämorrhagische Komponente dieser Läsionen nachgewiesen werden. Die Sonographie ist in dieser Phase nicht spezifisch. In der duktalen Phase (stationäre Phase während der Invasion der Gallenwege durch den Parasiten) können in der CT zentrale Cholestasezeichen demonstriert werden. Die Sonographie ist in dieser Phase diagnostisch führend: Sie kann den direkten Nachweis eines „mobilen“ Wurms liefern. Histomorphologisch bestehen die hypodensen Leberherde aus eosinophilen Granulomen mit hämorrhagischer Umwandlung (Migrationsweg des Parasiten) (Han et al., Invest Radiol 1999; 34 (2) und Richter et al., Trop Med Int Health 1999; 4: 774).

Die klinischen Symptome der Fasciolose sind insgesamt unspezifisch und bei fehlender Kenntnis der Veränderungen in den bildgebenden Verfahren täuschen die hepatischen und extrahepatischen Spuren des Parasiten leicht ein Malignom vor.
In der Literatur findet sich ca. ein Dutzend Fallberichte, die den computertomographischen Aspekt bei Leberbefall durch $F$. hepatica beschreiben: Hier stehen unscharf begrenzte, vor allem peripher im rechten Leberlappen lokalisierte kleine hypodense konfluierende Läsionen, die z.T. straßenförmig angeordnet sind, im Vordergrund.

Die Morphologie und Lokalisation der Leberläsionen unserer Patientin sind charakteristisch für die Fasciolose und auf die hepatische Migration zurückzuführen. Die beschriebene tubuläre Struktur im distalen Ductus choledochus und in der Gallenblase mit konsekutiver Dilatation der Gallenwege entsprach dem wandernden Leberegel selbst.

\section{Springende Punkte: „Fasciolose“}

- Aufenthalt in einem Entwicklungsland

- Oberbauchschmerzen

- Eosinophilie

- gruppierte hypodense Leberherde

- mobile cholangioläre RF

K. Brenner-Maucher, U. Szeimies, M. Mannstadt, M. Gross, München 